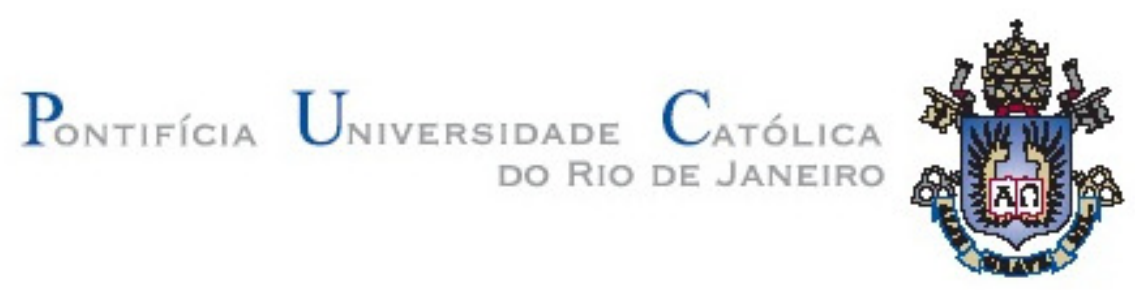

Jackeline Rosemery Castañeda Huertas

Procedimentos de Análise Não-linear para Previsão de Resposta Sísmica em Geoestruturas

Tese de Doutorado

Tese apresentada ao Programa de Pósgraduação em Engenharia Civil da PUC-Rio como requisito parcial para obtenção do título de Doutor em Engenharia Civil.

Orientador: Prof. Celso Romanel

Volume I

Rio de Janeiro

Junho de 2012 


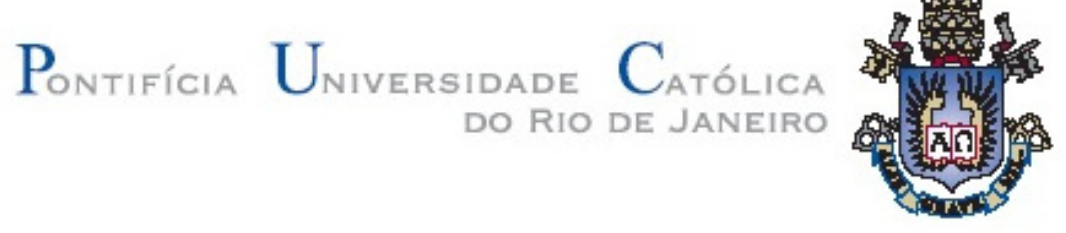

Jackeline Rosemery Castañeda Huertas

\section{Procedimentos de Análise Não-linear para Previsão de Resposta Sísmica em Geoestruturas}

Tese apresentada como requisito parcial para obtenção do título de Doutor pelo Programa de Pós-graduação em Engenharia Civil da PUCRio. Aprovada pela Comissão Examinadora abaixo assinada.

Prof. Celso Romanel Orientador Departamento de Engenharia Civil - PUC-Rio

Prof. Paulo Batista Gonçalves Departamento de Engenharia Civil - PUC-Rio

Prof. Pedricto Rocha Filho Departamento de Engenharia Civil - PUC-Rio

Dr. Nelson Inoue GTEP- PUC-Rio

Prof. Andréa Abreu Diniz de Almeida Universidade Federal Fluminense

Prof. Maria Cascão Ferreira de Almeida Universidade Federal do Rio de Janeiro

Prof. José Eugênio Leal Coordenador Setorial do Centro Técnico Científico - PUC-Rio

Rio de Janeiro, 27 de junho de 2012 
Todos os direitos reservados. É proibida a reprodução total ou parcial do trabalho sem autorização da universidade, da autora e do orientador.

\section{Jackeline Rosemery Castañeda Huertas}

Graduou-se em Engenharia Civil pela Universidad Privada Antenor Orrego (UPAO), de Trujillo - Peru, em 1999, tendo exercido a profissão de engenheira civil na cidade de Iquitos - Peru até 2003. Ingressou em 2004 no curso de Mestrado e em 2007 prosseguiu no curso de Doutorado em Engenharia Civil da Pontifícia Universidade Católica do Rio de Janeiro, na área de Geotecnia, desenvolvendo investigações na linha de pesquisa em Geomecânica Computacional.

Ficha Catalográfica

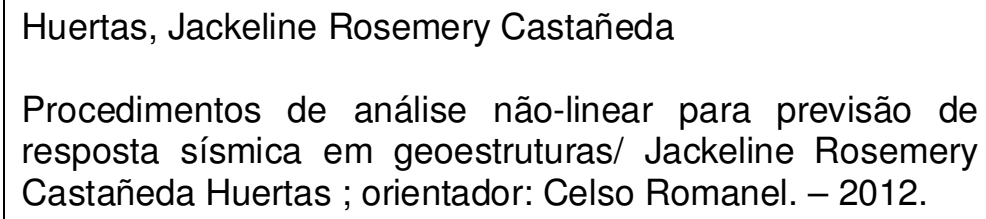
resposta sísmica em geoestruturas/ Jackeline Rosemery Castañeda Huertas ; orientador: Celso Romanel. - 2012.

2v. il; $29,7 \mathrm{~cm}$.

Tese (Doutorado em Engenharia Civil)-Pontifícia Universidade Católica do Rio de Janeiro, Rio de Janeiro, 2012.

Inclui bibliografia

1. Engenharia civil - Teses. 2. Modelagem numérica. 3. Liquefação dinâmica em solos. 4. Análise sísmica. 5. Estruturas nucleares. I. Romanel, Celso. II. Pontifícia Universidade Católica do Rio de Janeiro. Departamento de Engenharia Civil. III. Título. 
Aos meus amados pais, Lydia Norma e Henry Alejandro. 


\section{Agradecimentos}

Gostaria de agradecer ao corpo docente do Departamento de Engenharia Civil da Pontifícia Universidade Católica do Rio de Janeiro. Em especial ao professor Celso Romanel pela presente orientação e formação ao longo de todo esse tempo de trabalho.

Aos membros da Banca Examinadora, Paulo Batista Gonçalves, Pedricto Rocha Filho, Andréa Abreu Diniz de Almeida, Maria Cascão Ferreira de Almeida, Nelson Inoue e Celso Romanel, pelas valiosas contribuições buscando o aperfeiçoamento deste trabalho.

Um agradecimento especial aos meus colegas e amigos pelas discussões e contribuições ao longo desta pós-graduação, Carmen Ayquipa, Fanny Herrera, Gricel Portillo, Flávia Falcão, Thaís Abreu, Vivian Marchesi, Mariana Benessuiti, Eliot Pezo, Fabrício Zuleta, Ramiro Piedra, Carlos Soto, Wagner Nahas, Julio Bizarreta, Danilo Ramos, Martin Purizaga, Christian Elescano e Frank Pérez. Da mesma forma para Zenón Aguilar Bardales, José Huertas Polo, Ana Paula Martins Názario e à dona Vane Serra Pedroso.

Às prezadas amigas do Departamento de Engenharia Civil, Rita de Cássia do Nascimento Leite, Paula Enoy Nobrega Teixeira e Maria de Fátima de Castro Dinoá.

À Pontifícia Universidade Católica do Rio de Janeiro (PUC-Rio), à Fundação de Amparo à Pesquisa do Estado do Rio de Janeiro (FAPERJ), ao Conselho Nacional de Desenvolvimento Científico e Tecnológico (CNPq) e à Coordenação de Aperfeiçoamento de Pessoal de Nível Superior (CAPES) pelas respectivas concessões de bolsa de estudos que viabilizaram minha permanência durante o curso. 


\section{Resumo}

Huertas, Jackeline Rosemery Castañeda; Romanel, Celso (Orientador). Procedimentos de análise não-linear para previsão de resposta sísmica em geoestruturas. Rio de Janeiro, 2012. 348 p. Tese de Doutorado Departamento de Engenharia Civil, Pontifícia Universidade Católica do Rio de Janeiro.

O estudo do comportamento de solos sob carregamento sísmico é de grande importância para o projeto de geoestruturas situadas em regiões de alta atividade sísmica, como nos países andinos ao longo da borda da placa tectônica sul-americana. No Brasil, localizado no interior desta placa, onde eventos sísmicos são menos frequentes e de menor magnitude, um projeto dísmico detalhado é necessário para algumas obras de engenharia de alta importância como centrais nucleares. $\mathrm{O}$ objetivo principal desta dissertação é investigar o comportamento sísmico de geoestruturas, descrevendo e discutindo os vários pontos que devem ser cuidadosamente considerados pelos projetistas sob ponto de vista da engenharia geotécnica. Em particular, o comportamento de um cais para submarinos nucleares, projetado para ser construído no litoral do estado do Rio de Janeiro - Brasil, é analisado considerando aspectos relacionados com o potencial de liquefação dinâmica e a resposta sísmica dos solos em termos de histórias de aceleração, espectros de resposta e deslocamentos permanentes. O modelo constitutivo UBCSAND foi usado para representar a resposta de liquefação de areais saturas sob carregamento cíclico e alguns programs computationais (FLAC, SHAKE2000) foram empregados para calcular as respostas esperadas das geoestruturas.

\section{Palavras - chave}

Modelagem numérica; liquefação dinâmica em solos; resposta sísmica de geoestruturas. 


\section{Abstract}

Huertas, Jackeline Rosemery Castañeda; Romanel, Celso (Advisor). Procedures for nonlinear analysis prediction of seismic response of geostructures. Rio de Janeiro, 2012. 348p. DSc. Thesis - Departamento de Engenharia Civil, Pontifícia Universidade Católica do Rio de Janeiro.

The study of the soil behaviour under seismic loading is of great importance for the design of geostructures situated in regions of high seismic activity such as in the Andean countries along the border of the South American tectonic plate. In Brazil, situated in the interior of this plate, where seismic events are less frequent and of the smaller magnitude, a detailed seismic design is necessary for some engineering works of high importance such as nuclear power plants. The main objective of this thesis is to investigate the seismic behaviour of geostructures, describing and discussing the several points that should be carefully considered by the designers under the geotechnical engineering standpoint. In particular, the behavior of a nuclear wharf planned to be built on the seashore of the state of Rio de Janeiro - Brazil, is analyzed considering aspects related to the potential of dynamic soil liquefaction and the seismic response of soils in terms of accelerations histories, response spectra and permanent displacements. The UBCSAND constitutive model was used to represent the liquefaction response of saturated sands under cyclic loading and some computational programs (FLAC, SHAKE2000) were used in order to calculate the expected response of the geostrucures.

\section{Keywords}

Numerical model; soil dynamic liquefaction; seismic response of geostructures. 


\section{Sumário}

1 Introdução $\quad 27$

$\begin{array}{ll}1.1 \text { Abordagem desta pesquisa } & 27\end{array}$

1.1.1 Estudo de caso 31

1.2 Motivação e objetivos da tese 31

1.3 Organização da tese $\quad 33$

2 Comportamento das Areias Sob Carregamento Cíclico 34

2.1 Introdução 34

2.2 Mecanismo da dilatância 36

2.3 Comportamento monotônico e cíclico das areias saturadas $\quad 40$

2.3.1 Carregamento monotônico $\quad 42$

$\begin{array}{ll}\text { 2.3.2 Carregamento cíclico } & 49\end{array}$

2.4 Resistência 53

2.5 Rotação das tensões principais $\quad 55$

2.6 Ensaios dinâmicos para a obtenção das propriedades dinâmicas dos solos $\quad 60$

3 Liquefação e Pós-Liquefação Dinâmica em Solos Saturados 64

3.1 Introdução à liquefação dinâmica 64

3.2 Parâmetros de estado na abordagem do estado crítico 67

$\begin{array}{ll}3.3 \text { Mecânica da Liquefação } & 71\end{array}$

3.3.1 Mecanismos de Início de Liquefação $\quad 72$

3.3.1.1 Fluxo por liquefação

3.3.1.2 Mobilidade cíclica $\quad 74$

3.3.2 Susceptibilidade à liquefação $\quad 76$

$\begin{array}{ll}3.4 \text { Avaliação do início de liquefação } & 78\end{array}$

3.4.1 Caracterização baseada em ensaios de laboratório $\quad 85$

3.4.2 Caracterização baseada em ensaios In Situ $\quad 87$

3.4.2.1 Ensaio de penetração padrão (SPT) 89

3.4.2.2 Ensaio de penetração de cone (CPT) 92

$\begin{array}{ll}\text { 3.4.2.3 Velocidade das ondas cisalhantes, } v_{s} & 94\end{array}$

$\begin{array}{ll}\text { 3.4.3 Fator de segurança contra liquefação } & 97\end{array}$

3.5 Resistência residual mobilizada 98

3.6 Método simplificado de análise de liquefação para taludes de solos
(Olson\& Stark)

3.6.1 Análise de suscetibilidade à liquefação 104

3.6.2 Análise de iniciação de liquefação 104

3.6.3 Análise de estabilidade pós-liquefação 106

3.7 Análise de estabilidade pseudo-estática 107

$\begin{array}{ll}\text { 3.7.1 Coeficiente sísmico, k } & 109\end{array}$

$\begin{array}{ll}\text { 3.7.2 Parâmetros de resistência } & 112\end{array}$ 
3.7.3 Análise pós-sismo 113

$\begin{array}{ll}\text { 3.7.4 Comentários finais } & 114\end{array}$

4 Aspectos da Análise Sísmica em Geoestruturas 116

4.1 Introdução 116

4.2 Conceitos básicos de sismicidade $\quad 116$

$\begin{array}{ll}\text { 4.2.1 Sismicidade e tectônicas das placas } & 117\end{array}$

4.2.1.1 Regiões intraplacas 118

$\begin{array}{lr}\text { 4.2.1.2 Ondas sísmicas } & 120\end{array}$

4.2.1.3 Atenuação das ondas sísmicas 123

$\begin{array}{ll}\text { 4.2.2 Sismos } & 124\end{array}$

4.2.2.1 Localização de um sismo $\quad 125$

4.2.2.2 Grandeza de um sismo: magnitude e intensidade 126

$\begin{array}{lr}\text { 4.2.2.3 Energia de um terremoto } & 129\end{array}$

4.2.3 Efeitos dos sismos 133

4.3 Movimento do terreno 133

4.3.1 Caracterização e estimativa dos parâmetros do movimento do terreno 133

4.3.2 Amplitude do movimento do terreno 134

4.3.3 Conteúdo de frequências 136

4.3.4 Duração do movimento 139

4.3.5 Relações de prognóstico para a estimativa dos parâmetros de movimento $\quad 140$

4.4 Avaliação da ameaca sísmica 144

4.4.1 Identificação e caracterização da fonte sísmica 146

$\begin{array}{ll}\text { 4.4.2 Sismo de magnitude máxima } & 148\end{array}$

$\begin{array}{ll}\text { 4.4.3 Relações e intervalo de recorrência } & 148\end{array}$

$\begin{array}{ll}\text { 4.4.4 Relações de prognóstico } & 150\end{array}$

4.4.5 Curva de ameaça sísmica $\quad 151$

4.5 Efeitos do sítio local 153

4.6 Carregamento sísmico: espectros e sismos de projeto 156

4.6.1 Métodos para determinação de espectros de projeto 157

4.6.2 Métodos para geração de acelerogramas artificiais 164

$\begin{array}{ll}\text { 4.7 Processamento de registros sísmicos } & 168\end{array}$

4.8 Caracterização das propriedades dinâmicas: módulo de cisalhamento e
razão de amortecimento

$\begin{array}{ll}\text { 4.8.1 Módulo cisalhante } & 172\end{array}$

4.8.2 Razão de amortecimento 177

$\begin{array}{lr}4.9 \text { Amortecimento } & 179\end{array}$

4.9.1 Amortecimento de Rayleigh $\quad 180$

4.9.2 Amortecimento histerético 183

5 Métodos de Análise Sísmica $\quad 185$

5.1 Aplicação dos métodos de análise sísmica $\quad 185$

5.2 Análise de reposta sísmica do terreno de campo livre 186

5.2.1 Aproximação linear equivalente da resposta não-linear 189

5.2.2 Abordagem não-linear unidimensional 191

5.2.3 Análise de deconvolução e/ou convolução 193

5.3 Análises simplificadas de resposta sísmica por deslocamento 196

5.4 Análise sísmica não-linear 197

5.4.1 Método das diferenças finitas (FLAC) 198 
$\begin{array}{ll}\text { 5.4.2 Passo de tempo dinâmico } & 204\end{array}$

5.4.3 Considerações da modelagem dinâmica 208

5.4.4 Aferição das propriedades dinâmicas 213

$\begin{array}{ll}\text { 5.4.5 Fator de segurança pós-sismo } & 215\end{array}$

$\begin{array}{ll}\text { 5.5 Comentários finais } & 216\end{array}$

6 Modelos Constitutivos Cíclicos para Solos Arenosos 218

6.1 Relações tensão-deformação para solos 218

6.2 Uma breve descrição dos elementos básicos da teoria elasto-plástica
incremental

6.3 Modelo UBCSAND (Byrne et al., 1995) 226

6.3.1 Descrição das assunções e características do modelo 227

\begin{tabular}{ll} 
6.3.2 Resposta elástica & 230 \\
\hline & 230
\end{tabular}

6.3.3 Resposta plástica 231

7 Estudo de Caso: Análise dos Solos de Fundação e Maciço de

$\begin{array}{ll}7.1 \text { Local e condições da região em estudo } & 235\end{array}$

$\begin{array}{ll}\text { 7.1.1 Aspectos geológicos e tectônicos } & 237\end{array}$

$\begin{array}{ll}7.2 \text { Análise do nível de ameaça sísmica } & 238\end{array}$

7.3 Descrição do sismo de projeto adotado nas análises sísmicas 240

7.4 Análise de deconvolução do sismo de projeto 242

$\begin{array}{ll}7.5 \text { Processamento do registro sísmico } & 243\end{array}$

7.6 Verificação da resposta do terreno de campo livre 245

$\begin{array}{ll}7.7 \text { Análise de liquefação sísmica } & 247\end{array}$

$\begin{array}{ll}7.8 \text { Análise de resposta sísmica } & 250\end{array}$

$\begin{array}{ll}\text { 7.8.1 Modelo constitutivo } & 253\end{array}$

$\begin{array}{ll}\text { 7.8.2 Amortecimento } & 253\end{array}$

7.8.3 Parâmetros geotécnicos $\quad 254$

7.8.4 Condições de contorno na modelagem numérica com o programa FLAC $\quad 254$

\begin{tabular}{ll} 
7.8.5 Resultados das análises & 258 \\
\hline & 258
\end{tabular}

\begin{tabular}{ll} 
7.8.5.1 Seção A-A' & 258 \\
\hline & 280
\end{tabular}

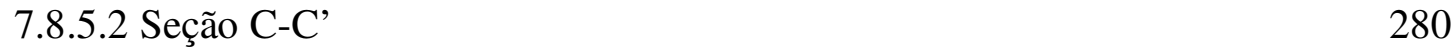

8 Conclusões e Sugestões 299

$\begin{array}{ll}8.1 \text { Conclusões } & 299\end{array}$

$\begin{array}{ll}8.2 \text { Sugestões } & 301\end{array}$

Referências Bibliográficas $\quad 303$

A Anexo $\quad 319$

A.1. Procedimento simplificado para a avaliação de tensões induzidas $\begin{array}{ll}\text { por terremotos } & 319\end{array}$

A.2. Cálculo da resistência $N_{1-60}$ corrigida para areia limpa (teor de finos) 322

A.2.1. Youd et al. (2001) 323

A.2.2. Idriss \& Boulanger (2004) 323

A.2.3. Outras correções aplicadas à resistência à penetração, N1-60 324

A.3. Ajustes e correções na resistência à penetração do cone 325

A.3.1. Ajuste e normalização da resistência de ponta do cone 326

A.3.2. Correção por areia limpa da resistência de ponta 326

A.3.3. Correção da resistência à penetração do cone por camadas de solos
fofos (ou moles) 
A.4. Fatores de escala de magnitude de terremotos (MFS) 330

A.5. Procedimentos alternativos dos fatores de correções $K_{\sigma}$, (por pressões de sobrecarga), $K_{\alpha}$ (por tensão cisalhante estática) $\quad 334$

A.5.1. Fator de correção $K_{\sigma} \quad 334$

$\begin{array}{ll}\text { A.5.2. Fator de correção } K_{\alpha} & 337\end{array}$

$\begin{array}{lr}\text { A.6. Razão de poropressão } & 339\end{array}$

B Anexo 341

B.1. Coeficientes das equações de atenuação do Toro et al. (1997) 341

B.2. Resumo de leis de atenuação para diferentes sítios 343

$\begin{array}{ll}\text { C Anexo } & 344\end{array}$

C.1. Geração de sismos artificiais no domínio do tempo 344 


\section{Lista de Figuras}

Figura 2.1- (a) Cisalhamento de camadas de materiais, de discos circulares, fracamente empacotados; (b) Cisalhamento de camadas de materiais, de discos circulares, densamente empacotados; (c) mudança de volume durante o cisalhamento de camadas de materiais, de discos circulares, fracamente e densamente empacotados. (Wood, 2004).

Figura 2.2- Superfícies de cisalhamento inclinadas causando dilatância $\psi$ e consequentes ângulos de atrito mobilizado $\phi_{\mathrm{m}}$.(Wood, 2004).

Figura 2.3- Relações entre razão de tensões normal e cisalhante, $\eta=t / \sigma$, e razão de incremento de deformações, $-\left(\Delta \varepsilon_{v} / \Delta \gamma\right)$, para a areia de Otawa em ensaios de cisalhamento simples drenado (dados básicos obtidos a partir de Vaid et al., 1981) (Puebla, 1999).

Figura 2.4- Trajetórias de tensões para carregamento drenado monotônico com p' e carregamento não drenado (cisalhamento a volume constante) de areias saturadas fofas-a partir do-estado crítico e densas-a partir do-estado crítico (Idriss \& Boulanger, 2008).

Figura 2.5- Resposta ao carregamento monotônico de amostras fofas $(\mathrm{DR}=38 \%)$ e densas $(\mathrm{DR}=100 \%)$ da areia de do Rio Sacramento em ensaios de compressão triaxial drenado, como se mostra nos gráficos (a) e (b) respectivamente (Idriss \& Boulanger, 2008, adaptado de Lee \& Seed, 1967). Figura 2.6 - Classificação de comportamento não drenado de solos arenosos baseados em fenômenos de dilatância e amolecimento (Ishihara, 1996).

Figura 2.7- Reposta ao carregamento monotônico da areia de Toyoura saturada em ensaios de compressão triaxial CIU (Ishihara, 1993).

Figura 2.8- Estado quase permanente (QSS) e comportamento quase permanente de areia Toyoura muito fofa em ensaios de compressão triaxial CIU (Ishihara 1993).

Figura 2.9 - Mecanismo de geração de poropressão durante o carregamento (Idriss \& Boulanger, 2008)

Figura 2.10 - Resposta da areia de Sacramento ao carregamento triaxial cíclico não drenado (ensaios de Boulanger \& Truman, 1996)

Figura 2.11 - Resultados de ensaios de rotação contínua: (a) variação da direção das tensões principais e razão de tensões, e (b) deformação acumulada principal maior em areias densas (Arthur et al., 1980).

Figura 2.12 - Resultados de ensaios de rotação de tensões em areia fofa de Toyoura (Ishihara \& Towhata, 1983).

Figura 2.13 - Resposta das trajetória de tensões e deformações não drenada da areia de Rio Fraser testado ao longo das diferentes trajetórias de tensões em um torsional hollow cylinder (after Vaid \& Eliadorani, 1998) 
Figura 2.14 - Coeficiente de poropressão versus direções da tensão principal maior (Kumruzzaman \& Yin (2010).

Figura 2.15- Métodos sísmicos para a determinação de perfis da rigidez do solo com a profundidade (McDowell et al., 2002).

Figura 3.1 - Esquema de resposta não drenada de um solo arenoso, contrativo e saturado (Olson, 2001).

Figura 3.2 - Três casos de mobilidade cíclica mostrado no espaço de trajetória de tensões: (a) sem reversão de tensões e tensões cisalhantes cíclicas e estáticas combinadas menores do que a resistência no estado permanente; (b) sem reversão de tensões e períodos temporários onde as tensões cisalhantes cíclicas e estáticas combinadas excederam a resistência no estado permanente; e (c) com reversão de tensões e tensões cisalhantes estáticas e cíclicas combinadas menores do que a resistência no estado permanente (Kramer, 1996).

Figura 3.3 - Razão de tensões cíclicas requerida para alcançar o início da liquefação $\left(r_{u}=100 \%\right)$, de ensaios da mesa vibratória por De Alba et al. (1976) (adaptado por Idriss \& Boulanger, 2008).

Figura 3.4- (a) Definição do índice de parâmetro de estado relativo, $\xi_{R}$ (Boulanger, 2003), (b) CRR versus $\xi_{R}$ para amostras reconstruídas da Areia de Fraser Delta (Boulanger, 2003) (Idriss \& Boulanger, 2008).

Figura 3.5- (a) Curva base para areias limpas para ensaios SPT, para magnitude de terremotos de 7,5 com dados extraídos de casos históricos com ocorrência de liquefação (Youd et al., 2001, modificado a partir de Seed et al., 1985); (b) Casos Históricos de SPT para solos sem coesão com $\mathrm{FC} \geq 35 \%$, curvas da NCEER (1997) e a recomendada por Idriss \& Boulanger (2004).

90

Figura 3.6- Curvas recomendadas para o cálculo da CRR para terremotos de magnitude $\mathrm{M}_{\mathrm{w}}=7,5$ elaboradas a partir de ensaios CPT baseadas em dados de liquefação (NCEER 1997).

Figura 3.7 - Curvas recomendadas para cálculo da CRR a partir de medidas de $\mathrm{V}_{s 1}$ para solos limpos não cimentados baseados em dados históricos (Andrus \& Stokes, 2000).

Figura 3.8 - Zona esperada a ser liquefeita determinada pela comparação das tensões cíclicas induzidas por terremotos, com as resistências cíclicas do solo (Idriss \& Boulanger, 2008).

Figura 3.9- Características do comportamento não drenado de areias fofas (Ishihara, 1996).

Figura 3.10- Análise de suscetibilidade à liquefação (Olson, 2003)

100

Figura 3.11 - Razão de resistência de escoamento para análise de iniciação de liquefação (Olson, 2003)

Figura 3.12 - Razão de resistência liquefeita para análise pós- liquefação (Olson, 2003).

106

Figura 3.13- Conceitualização do método pseudo-estático (Franklin \& Chang, 1977)

Figura 3.14- Mapeamento da aceleração sísmica horizontal característica no brasil para terrenos de classe B (Rocha) (NRB 15421/2006). 
Figura 4.1- A Litosfera, a camada mais superficial da Terra, está dividida em grandes porções, chamadas de PLACAS LITOSFÉRICAS (USGS, Washington, 2001).

Figura 4.2 - Esquematização de movimentos interplacas 118

Figura 4.3 - Sismicidade natural do Brasil - Universidade de Brasília. $\quad 119$

Figura 4.4 - Principais ondas sísmicas com propósitos de engenharia (Kuroiwa, 2002), apud Gamarra (2009).

121

Figura 4.5 - Reflexão e Refração: (a) de ondas P ou SV; (b) de ondas SH (Adaptado de Kramer, 1996).

Figura 4.6 - Decréscimo de amplitude de ondas sísmicas com a distância $(r)$ a partir da fonte (amortecimento geométrico), (Woods, 1968) apud Towhata (2008).

Figura 4.7 - Elementos para descrição da localização de um sismo

Figura 4.8 - Ilustração da relação entre magnitude e a escala de saturação de várias magnitudes: $\mathrm{M}_{\mathrm{W}}$ (Magnitude do Momento Sísmico), $\mathrm{M}_{\mathrm{L}}$ (Magnitude Local Richter), $\mathrm{M}_{\mathrm{S}}$ (Magnitude de ondas superficiais), $\mathrm{m}_{\mathrm{b}}$ (Magnitude de ondas de corpo de período curto), $\mathrm{m}_{\mathrm{B}}$ (Magnitude de ondas de corpo de período longo) e $\mathrm{M}_{\mathrm{JMA}}$ (Magnitude da Agência Meteorológica Japonesa) (Kramer, 1996).

Figura 4.9 - Energia relativa de vários fenômenos humanos e naturais (Johnston, 1990) apud Kramer (1996).

130

Figura 4.10 - Principais fatores que influenciam os efeitos dos sismos: fonte, trajetória percorrida do sismo e características do sítio. Ponto A equivalente a solo, amplificando a baixas frequências, e ponto $\mathrm{B}$, equivalente a rocha, amplificando a altas frequências.

Figura 4.11 - Espectros de resposta de projeto independentes do tipo de solo (Almeida, 1997).

Figura 4.12 - (a) Espectro de resposta da componente N-S do terremoto El Centro (18-maio-1940) e (b) Espectro de resposta dependente de $a_{\max }$, $v_{\max } \mathrm{e}$ $\mathrm{d}_{\max }$. (Clough \& Pezien, 1993).

Figura 4.13 - Espectros de resposta de projeto $\left(\mathrm{Sa} / \mathrm{a}_{\mathrm{gs} 0}\right)$ em função do período T(s) da NBR 15421.

Figura 4.14 - Espectros de resposta de projeto suavizado (Adaptado de Almeida 1997).

Figura 4.15 - Geração artificial de movimentos de terreno (adaptado de Kramer, 1996).

Figura 4.16 - Exemplo de uma função sintética no tempo gerada no domínio da frequência (modificado de Kramer, 1996).

Figura 4.17 - Ajuste de Linha base. (after Carreño et al.,1999).

Figura 4.18 - Módulo de cisalhamento secante, $\mathrm{G}_{\mathrm{sec}}$, e módulo de cisalhamento tangente, $\mathrm{G}_{\mathrm{tan}}$. (Kramer, 1996).

161

163

166

167

170

Figura 4.19 - Curvas de redução de módulos para solos granulares finos de diferentes plasticidades (After Vucetic \& Dobry, 1991).

172

Figura 4.20 - Variação da razão de amortecimento de solos granulares finos com a amplitude de deformação cisalhante cíclica e o índice de plasticidade (After Vucetic \& Dobry,1991). 
Figura 4.21 - Variação da razão de amortecimento crítica normalizada com a frequência angular. Nesta figura, a linha azul, $\alpha=0$, a linha vermelha, $\beta=0$, e a linha verde, o total.

181

Figura 4.22- Relações entre o laço histerético e a razão de amortecimento. (Kramer, 1996)

Figura 5.1 - Esquema para a nomenclatura da resposta do terreno: (a) solo sobrejacente; (b) sem solo sobrejacente (Kramer, 1996).

188

Figura 5.2 - Processo para obter a reposta do terreno em um perfil como o esquematizado em (a). Em (b) o registro sísmico de entrada na rocha, (c) é a transformada de Fourier do sismo de entrada. Em (d) a função de transferência. A série de Fourier do sismo na superfície do terreno foi calculada com o produto da função de transferência e série de Fourier do sismo na rocha. A inversão da série de Fourier produz a história no tempo das acelerações na superfície do terreno, mostrado em (e) (Kramer, 1996).

Figura 5.3 - Processo de deconvolução e amplificação (convolução) para registros de terremotos.

Figura 5.4 - Sismo de entrada para análises numéricas (Mejia \& Dawson, 2006).

194

Figura 5.5 - Processo de deconvolução condições de contorno a) base rígida e, b) base flexível (Mejia \& Dawson, 2006)

Figura 5.6 - Ciclo básico de cálculo explícito (Cundall, 2009).

199

Figura 5.7 - Elemento básico da malha de diferenças finitas: (a) elementos quadrangulares sobrepostos; (b) elementos triangulares típicos com vetores de velocidade; e, (c) vetor de forças nodais (Cundall, 2009).

200

Figura 5.8 - Modelagem do contorno silencioso implementado no FLAC (adaptado por Loayza (2009) de Cundall (2009).

Figura 5.9 - Malha de campo livre (free-field) utilizada na avaliação dinâmica no FLAC (adaptado por Loayza (2009).

212

Figura 5.10 - Condições de contorno aplicadas à geoestruturas no FLAC (adaptado por Loayza (2009).

Figura 6.1- Superfície de escoamento de forma de uma bala como proposta por Drucker et al. (1955) para condições triaxiais.

Figura 6.2 - Lei de fluxo usada no UBCSAND. Deformações cisalhantes induzem contração volumétrica sob $\varphi_{\mathrm{cv}}$ ou $\varphi_{\mathrm{pt}}$; e sobre esta, induzem dilatância volumétrica (Naesgaard, 2011).

Figura 6.3 - Relação Hiperbólica Tensão-Deformação (adaptado de Park, 2005).

Figura 7.1 - Mapa Tectônico da região de estudo, mostrando as principais feições tectônicas da região Sudeste do Brasil e áreas vizinhas. PEM representa a Província Estrutural Mantiqueira, CSF o Craton de São Francisco, CDB o Cinturão de Dobramentos Brasília e BP a Bacia do Paraná. O triângulo vermelho representa o local de interesse do presente projeto. A legenda das unidades geológicas não se encontra aqui referenciado, recomenda-se procurar o mapa elaborado por Schobbenhaus et al. (1984). 
Figura 7.2 - Mapa da região que abrange a AII, mostrando as principais unidades tectônicas. Simplificado por Heilbron et al. (2000), Ferrari (1990) e Mohriak \& Barros (1990). Ferrari (2001).

Figura 7.3 - Acelerograma do sismo de projeto SH-2.

Figura 7.4 - Comparação do espectro de projeto do sismo SH-2 e da Norma Brasileira NBR15421/2006.

Figura 7.5 - Acelerograma do sismo SH-2, com deconvolução em rocha, aplicado em base flexível situada na cota $\mathrm{y}=-60 \mathrm{~m}$, corrigido por linha base e filtro de frequências passa-baixa com corte em $\mathrm{f}_{\mathrm{c}}=20 \mathrm{~Hz}$.

Figura 7.6 - Comparação dos espectros de resposta das acelerações horizontais antes e após a filtragem das acelerações horizontais superiores a $20 \mathrm{~Hz}$.

Figura 7.7 - Espectro de Fourier das acelerações.

Figura 7.9 - Esquema de localização dos furos de sondagem na região do enrocamento.

Figura 7.10 - Amplificação do sismo na localização da NSP-35 para o sismo SH-2.

Figura 7.11 - Amplificação do sismo na localização da SM-63 para o sismo SH-2.

Figura 7.12 - Planta de locação das seções A-A' e C-C'. numéricamente (linha cheia) para a variação da razão de módulos cisalhantes $\mathrm{G} / \mathrm{G}_{\max }$ com a deformação cisalhante para o caso de areias (curva limite superior de Seed \& Idriss, 1970), enrocamento (curva média de Seed et al., 1986) e silte/argilas (curva limite superior de Sun et al., 1988).

Figura 7.16 - Modelo discreto da seção A-A', formado por 11.023 elementos (zonas).

Figura 7.17 - Fatores de segurança estático do talude de enrocamento da seção A-A'.

Figura 7.18 - Distribuição dos excessos de poropressão no tempo $t=15 \mathrm{~s}$ (final do sismo) na seção A-A'. (unidade Pa).

Figura 7.19 - Distribuição dos valores da razão de poropressão ao final do sismo $(\mathrm{t}=15 \mathrm{~s})$ na seção A-A'.

Figura 7.20 - Indicação de áreas (em vermelho) onde $r_{u} \geq 1$ ao longo dos 15 s do sismo.

Figura 7.21 - Indicação de áreas (em vermelho) onde $r_{u} \geq 1$ ao final do sismo $(\mathrm{t}=15 \mathrm{~s})$.

Figura 7.22 - Localização dos pontos de controle para análise da variação no tempo da razão de poropressão $r_{u}$ na seção A-A'.

Figura 7.23 - Distribuição dos deslocamentos horizontais ao final do sismo $(\mathrm{t}=15 \mathrm{~s})$. Unidades em $\mathrm{m}$.

Figura 7.24 - Malha distorcida da seção $A$ - $A^{\prime}$ ao final do sismo $(t=15 \mathrm{~s})$. 
Figura 7.25 - História da razão de poropressão $r_{u}$ durante a ocorrência do sismo em pontos de controle na seção A-A'.

Figura 7.26 - Pontos de controle das histórias de aceleração horizontal, espectros de resposta e histórias de deslocamento horizontal na seção A-A'.

Figura 7.27- Histórias de aceleração horizontal em pontos de controle situados na superfície da seção A-A'.

Figura 7.28 - Histórias de aceleração horizontal em pontos situados no interior do maciço de solo e na base do modelo da seção A-A'.

Figura 7.29 - Espectros de resposta da aceleração horizontal em diferentes pontos de controle situados na superfície da seção A-A'.

Figura 7.30 - Espectros de resposta da aceleração horizontal em diferentes pontos de controle situados no interior do maciço de solo e na interface solo-rocha.

Figura 7.31 - Localização das colunas de solo para comparação dos resultados obtidos com os programas SHAKE e FLAC na seção A-A'.

Figura 7.32 - Comparação dos espectros de resposta calculados com os programas SHAKE e FLAC na seção A-A'.

Figura 7.33 - Histórias de deslocamento horizontal em diferentes pontos de controle situados na superfície da seção A-A'.

Figura 7.34 - Histórias de deslocamento horizontal em diferentes pontos de controle situados no interior do maciço de solo (seção A-A').

Figura 7.35 - Distribuição de deslocamentos horizontais no tempo $t=6.5 \mathrm{~s}$ (unidade $\mathrm{m}$ ).

Figura 7.36 - Distribuição de deslocamentos horizontais no tempo $t=15 \mathrm{~s}$ (unidade $\mathrm{m}$ ).

Figura 7.37 - Histórias de tensão e de poropressão no ponto $\mathrm{H}$ (convenção de sinais da Resistência dos materias).

Figura 7.38 - História de deformação cisalhante máxima em diferentes pontos de controle da seção A-A'.

Figura 7.39 - Distribuição das deformações cisalhantes máximas no tempo $\mathrm{t}=6.5 \mathrm{~s}$

Figura 7.40 - Distribuição das deformações cisalhantes máximas no tempo $\mathrm{t}=15 \mathrm{~s}$.

Figura 7.41 - Posição dos eixos das estacas E-1, E-2, E-3, E-4, E-5 com pontas situadas na cota $\mathrm{y}=-42 \mathrm{~m}$ ( seção $\mathrm{A}-\mathrm{A}^{\prime}$ ).

Figura 7.42 - Deslocamentos horizontais ao longo dos eixos das "estacas", relativos às pontas situadas na cota $\mathrm{y}=-42 \mathrm{~m}$, ao final do sismo (deslocamentos permanentes) e deslocamentos máximos durante o sismo. Os maiores deslocamentos na parte superior da "estaca 1" são devidos ao movimento do talude de enrocamento. (Figura 7.44).

Figura 7.43 - Modelo discreto da seção C-C', formado por 11.325 elementos (zonas).

Figura 7.44 - Fatores de segurança estática do talude de enrocamento da seção C-C'

Figura 7.45 - Distribuição dos excessos de poropressão no tempo $t=15 \mathrm{~s}$ (final do sismo) na seção C-C'. (unidade Pa) 
Figura 7.46 - Distribuição da razão de poropressão no tempo $t=15$ s (final do sismo) na seção C-C'.

Figura 7.47 - Indicação de áreas (em vermelho) onde $r_{u} \geq 1$ ao final do sismo $(\mathrm{t}=15 \mathrm{~s})$.

Figura 7.48 - Indicação de áreas (em vermelho) onde $r_{u} \geq 1$ ao longo dos 15 s do sismo.

Figura 7.49 - Localização dos pontos de controle para acompanhamento da variação da razão de poropressão $r_{u}$ no intervalo $0 \leq \mathrm{t} \leq 15 \mathrm{~s}$

282

Figura 7.50 - História da razão de poropressão $r_{u}$ durante a ocorrência do sismo em pontos de controle da seção C-C'

Figura 7.51 - Distribuição do deslocamento horizontal ao final do sismo $(\mathrm{t}=$ $15 \mathrm{~s}$ ). (unidades em $\mathrm{m}$ )

284

Figura 7.52 - Malha distorcida da seção C-C' ao final do sismo $(\mathrm{t}=15 \mathrm{~s})$.

284

Figura 7.53 - Pontos de controle das histórias de aceleração horizontal, espectros de resposta e histórias de deslocamento horizontal na seção C-C'.

Figura 7.54 - Histórias de aceleração horizontal em pontos de controle situados na superfície da seção C-C'.

Figura 7.55 - Histórias de aceleração horizontal em pontos de controle situados no interior do maciço de solo da seção C-C'.

Figura 7.56 - Espectros de resposta em diferentes pontos de controle situados na superfície da seção C-C'.

Figura 7.57 - Espectros de resposta em diferentes pontos de controle situados no interior do maciço de solo e na interface solo-rocha da seção CC'.

Figura 7.58 - Localização das colunas de solo para comparação dos resultados obtidos com os programas SHAKE e FLAC na seção C-C'.

286

287

288

289

Figura 7.59 - Comparação dos espectros de resposta calculados com os programas SHAKE e FLAC na seção C-C'.

Figura 7.60 - Histórias de deslocamento horizontal em diferentes pontos de controle na superfície da seção C-C'.

Figura 7.61 - Histórias de deslocamento horizontal em diferentes pontos de controle no interior do maciço de solo da seção C-C'.

Figura 7.62 - Distribuição de deslocamentos horizontais no tempo $t=7 \mathrm{~s}$ (unidade $\mathrm{m}$ ).

Figura 7.63 - Distribuição de deslocamentos horizontais no tempo $t=15 \mathrm{~s}$ (unidade $\mathrm{m}$ ).

Figura 7.64 - Histórias de tensão e de poropressão no ponto H (convenção de sinais da Resistência dos Materiais).

Figura 7.65 - Histórias de deformações cisalhantes máximas em diferentes pontos de controle da seção C-C'.

Figura 7.66 - Distribuição das deformações cisalhantes máximas no tempo $\mathrm{t}=7 \mathrm{~s}$

Figura 7.67 - Distribuição das deformações cisalhantes máximas no tempo $\mathrm{t}=15 \mathrm{~s}$

Figura 7.68 - Posição dos eixos das "estacas" E-1, E-2, E-3, E-4, E-5 com pontas situadas na cota 
Figura 7.69 - Deslocamentos horizontais ao longo dos eixos das "estacas", relativos às pontas situadas na cota $\mathrm{y}=-28 \mathrm{~m}$, ao final do sismo (deslocamentos permanentes) e deslocamentos máximos durante o sismo. Nota-se que os deslocamentos na parte superior do eixo da "Estaca" 1 são devidos aos movimento do talude (Figura 57).

Figura A. 1- Número de cíclos de tensão uniforme equivalente, $N_{e q}$, para terremotos de diferentes magnitudes (After Seed et al., 1975).

Figura A. 2 - Variação do coeficiente de redução de tensões, $r_{d}$, com a profundidade e magnitude de terremoto (Idriss, 1999).

Figura A. 3 - Fator de redução para a estimativa da variação das tensões cisalhantes cíclicas com a profundidade sob a superfície do terreno ou sob taludes suavizados (after Seed \& Idriss, 1971)

Figura A. 4 - Fator de correção das características do grão, $K_{c}$, para determinação da resistência CPT equivalente a areias limpas (Robertson \& Wride, 1998).

Figura A. 5 - Gráfico sobre o tipo de comportamento baseado em resultados de CPT proposto por Robertson (1990).

328

Figura A. 6 - Fator de correção para camadas finas, $K_{H}$, para a determinação da resistência do CPT para camadas de espessura equivalente (Robertson \& Fear, 1995).

Figura A. 7 - Fatores de escala da magnitude derivados de varios investigadores (reproduzido por Youd \& Noble, 1997 e adaptado por Youd et al., 2001)

Figura A. 8 - Curvas recomendadas por Hynes \& Olsen (1999) para estimativas mínimas e/ou conservativas de $\mathrm{K}_{\sigma}$ no caso de areias limpas, siltosas e pedregulhos para serem o usadas na prática da engenharia (Youd et al. (2001).

Figura A. 9 - Relações de $K_{\sigma}$ derivadas a partir de relações de $\xi_{R}$. Figura do dalo esquerdo de Boulanger \& Idriss, 2004), e figura do lado direito de Idriss \& Boulanger (2003).

Figura A. 10 - Variação do fator de correção, $K_{\alpha}$, com a razão de tensões normal/cisalhante iniciais. (after Seed \& Harder, 1990. H. Bolton Seed Memorial Symposium Proceedings, vol. 2) (adaptado por Kramer, 1996).

Figura A. 11 - Taxa de geração de poropressões de ensaios de cisalhamento simples cíclico. A linha tracejada foi gerada a partir da equação (A6.1) com $\alpha=0.7$ (After De Alba et al. 1975)

Figura A. 12 - Razão de excesso de poropressão versus $F S_{L}$ para materiais em condições sob a superficie do terreno, dados obtidos a partir de ensaios de laboratório (after Marcuson et al., 1990).

Figura C. 1 - Acelerograma com duração de 15s gerado a partir da FDEP (Figueiredo, 2004).

Figura C. 2 - Função Intensidade para um sismo com duração total de $15 \mathrm{~s}$ (Figueiredo, 2004). 
Figura C. 3 - Acelerograma após aplicado a Função Intensidade e correção de Linha base (Figueiredo, 2004).

Figura C. 4 - Diagrama de blocos que descreve o procedimento de geração de sismos artificiais (Notas de aula da disciplina CIV2122- Tópicos Especiais em Dinâmica de Estruturas, PUC-Rio, 2007). 


\section{Lista de Tabelas}

Tabela 1.1 - Alguns eventos importantes de deformação e liquefação induzida por terremotos. (adaptado e atualizado de Beaty, 2001)

Tabela 3.1 - Valores de Fatores de correção para a CRR, $c_{r}$ (adaptado de Kramer, 1996)

Tabela 3.2 - Comparação das vantagens e desvantagens dos vários ensaios de campo para avaliação da resistência à liquefação (Youd et al. 2001)

Tabela 3.3 - Coeficientes sísmicos (horizontais) recomendados (Melo \& Sarma, 2004)

Tabela 3.4 - Definição das zonas sísmicas no Brasil (NBR 15421/2006)

Tabela 4.1 - Velocidade das ondas sísmicas.

Tabela 4.2 - Comparação entre as escalas de Richter e Mercalli modificada.

Tabela 4.3 - Coeficientes $j$ da lei de atenuação de Joyner \& Boore (1988) para a Equação 4.16 (apud Kramer, 1996)

Tabela 4.4 - Definição de categorias do terreno pela NEHRP (Anbazhagan et al. 2007)

Tabela 4.5 - Classe de terreno usada na Norma Brasileira NBR 15421 (1996).

Tabela 4.6 - Fatores de Amplificação sísmica no solo usados na NBR 15421.

Tabela 4.7 - Exponente da razão de pre-adensamento, k (Kramer, 1996).

Tabela 4.10 - Relações empíricas de correlação entre $G_{\max }$ e parâmetros de ensaios in situ (modificado a partir de Kramer, 1996).

Tabela 4.11 - Efeitos das condições de carregamento e ambientais sobre os módulos de cisalhamento máximo, razão de módulos e razão de amortecimento de solos normalmente adensados e moderadamente sobreadensados (adaptado de Kramer, 1996).

Tabela 6.1 - Comparação de modelos plásticos típicos para areias sob carregamentos cíclicos e suas potencialidades (adaptado de Park, 2005).

Tabela 7.1 - Características dos sismos artificiais gerados pela empresa EGT Engenharia Ltda.

Tabela 7.2 - Amplificação do terreno.

Tabela 7.3 - Modelos constitutivos e parâmetros dos materiais da seção A-A'

Tabela 7.4- Modelos constitutivos e parâmetros dos materiais da seção C-C'

Tabela 7.5 - Valores máximos de aceleração horizontal calculados com os programas SHAKE e FLAC para a seção A-A'. 
Tabela 7.6 - Deslocamentos horizontais relativos à ponta das "estacas", ao longo dos respectivos eixos, ao final do sismo (deslocamentos permanentes) e deslocamentos máximos durante o sismo na seção A-A'

Tabela 7.7 - Valores máximos de aceleração horizontal calculados com os programas SHAKE e FLAC para a seção C-C'.

Tabela 7.8 - Deslocamentos horizontais relativos à ponta das "estacas", ao longo dos respectivos eixos, ao final do sismo (deslocamentos permanentes) e deslocamentos máximos durante o sismo na seção C-C'.

Tabela A. 1- Correlação do SPT (Modificado de Skempton, 1986) por Robertson \& Wride (1998) (Youd et al. 2001)

Tabela A. 2 - Valores de fatores de escala da magnitude definidos por vários investigadores (modificado de Youd et al., 2001)

Tabela B. 1 - Coeficientes das equações de atenuação

Tabela B. 2 - Valores da Incertezas aleatórias dependentes da magnitude $\left(\sigma_{a, \text { modelo }+\Delta \sigma}\right)$ para magnitudes críticas.

Tabela B. 3 - Valores da Incertezas aleatórias dependentes da distância $\left(\sigma_{a, \text { profundidade }+Q+\kappa}\right)$ para distâncias críticas. Norman A. e Shedlock, Kaye M., "Overview", Seismological Research Letters, 68-1, 1997) 


\section{Lista de Símbolos}

\begin{tabular}{|c|c|}
\hline a & Aceleração \\
\hline $\mathrm{ACU}$ & Ensaios triaxiais cíclicos não drenados adensados anisotropicamente \\
\hline BPT & Ensaio de penetração Becker \\
\hline$c$ & Coesão \\
\hline $\mathbf{C}$ & Matriz de amortecimento \\
\hline$C_{B}$ & Fator de correção por diâmetro do furo de sondagem do ensaio SPT \\
\hline$C_{E}$ & Fator de correção por energia do martelo do ensaio SPT \\
\hline$c_{n}$ & Amplitude do enésimo harmônico das séries de Fourier \\
\hline$C_{N}$ & Fator de correção por sobrecarga \\
\hline$C_{R}$ & Fator de correção por comprimento da haste \\
\hline$C_{S}$ & Fator de correção por amostradores com ou sem camisa \\
\hline CIU & Ensaios de compressão triaixial adensandos isotropicamente \\
\hline CPT & Ensaio de penetração de cone \\
\hline CSL & Linha do estado crítico \\
\hline CSR & Razão de tensões cíclicas ou demanda sísmica \\
\hline CRR & Razão de resistência cíclica ou resistência à liquefação do solo \\
\hline CSW & Análise ondas de superfície contínuas \\
\hline$D$ & Relação tensão-dilatância \\
\hline$d_{c}$ & Diâmetro do cone \\
\hline$D R$ & Densidade relativa \\
\hline$d x / d y$ & Inclinação de curva \\
\hline $\mathrm{E}$ & Módulo de Elasticidade \\
\hline$E$ & Energia do sismo \\
\hline $\bar{E}_{r}$ & Módulo tangencial da curva de descarregamento unidimensional \\
\hline$e$ & Índice de vazios \\
\hline$e_{s s}$ & Índice de vazios na condição de estado permanente \\
\hline $\mathrm{FC}$ & Teor de finos \\
\hline$f_{c}$ & Frequência de esquina \\
\hline$f_{\max }$ & Frequência de corte \\
\hline$F_{M(m)}$ & Função densidade de probabilidade de magnitude $\mathrm{m}$ \\
\hline$F S$ & Fator de segurança $\left(F S_{\text {estático }}\right)$ \\
\hline$F S_{L}$ & Fator de segurança contra a liquefação \\
\hline $\mathrm{FS}_{\text {flow }}$ & Fator de segurança contra a liquefação pós-liquefação \\
\hline$g$ & Aceleração gravitacional ou gravidade \\
\hline$G$ & Módulo de cisalhamento \\
\hline$G_{i}^{e}$ & Módulo de cisalhamento elástico \\
\hline$G_{i}^{p}$ & Módulo de cisalhamento plástico \\
\hline$G_{m n}$ & Módulo de cisalhamento tangente inicial máximo para o ciclo $\mathrm{n}$ \\
\hline$G_{m o}$ & Módulo de cisalhamento tangente inicial máximo \\
\hline$G_{(\omega)}$ & Espectro de potência ou função densidade espectro de potência \\
\hline$H_{1}, H_{2}, H_{3}, H_{4}$ & Constantes \\
\hline
\end{tabular}




\begin{tabular}{|c|c|}
\hline$I_{c}$ & Indicador do tipo de comportamento do solo para o ensaio CPT \\
\hline$I_{A}(t)$ & Função intensidade \\
\hline ICD & Ensaios triaxiais de compressão isotrópica drenada \\
\hline$k$ & Permeabilidade intrínseca ou coeficiente de mobilidade \\
\hline $\mathrm{k}$ & Coeficiente sísmico \\
\hline$k_{H}$ & Condutividade Hidráulica \\
\hline$K_{H}$ & Fator de correção do ensaio CPT para camadas \\
\hline $\mathbf{K}$ & Parcela de amortecimento proporcional à rigidez \\
\hline$K$ & Módulo de compressão volumétrica \\
\hline$K_{c}$ & Fator de correção do ensaio CPT por características de graõs \\
\hline$K^{e}$ & Módulo de deformação volumétrica na condição elástica \\
\hline$K_{o}$ & Coeficiente de empuxo em repouso \\
\hline$K c$ & Coeficiente de empuxo em condições de carregamento anisotrópico \\
\hline$K_{w}$ & Módulo de compressão volumétrica da água \\
\hline$k_{2}$ & Constante \\
\hline$K_{2, \max }$ & Parâmetro utilizado para estimar o módulo de cisalhamento \\
\hline$K_{m}$ & Constante \\
\hline$K \alpha$ & Fator de correção por tensão cisalhante estática \\
\hline$K_{\sigma}$ & Fator de correção por sobrecarga \\
\hline$K_{G}^{e}$ & Número do módulo de cisalhamento na condição elástica \\
\hline $\mathrm{L}$ & Onda Love \\
\hline $\mathbf{M}$ & Parcela de amortecimento proporcional à massa \\
\hline$[M]$ & Matriz de massa \\
\hline$M$ & Módulo tangente restrito da tensão efetiva \\
\hline MASW & Análise multicanais para ondas de superfície \\
\hline MMI & Intensidade Mercalli Modificada \\
\hline $\mathrm{m}_{\mathrm{b}}$ & Magnitude das ondas de corpo de período curto \\
\hline $\mathrm{m}_{\mathrm{B}}$ & Magnitude das ondas de corpo de período largo \\
\hline$M_{L}$ & Magnitude local \\
\hline $\mathrm{M}_{\mathrm{S}}$ & Magnitude de ondas superficiais \\
\hline $\mathrm{M}_{\mathrm{w}}$ & Magnitude do momento sísmico \\
\hline$m_{o}$ & Magnitude mínima \\
\hline$m_{\max }$ & Magnitude máxima \\
\hline$n$ & Porosidade \\
\hline & Razão de tensões mobilizadas \\
\hline$N_{B C}$ & Contagem do número de golpes do ensaio BPT \\
\hline$N_{\text {corr }}$ & Função de porcentagem de finos \\
\hline$N_{e q}$ & Número de ciclos de tensões uniformes equivalente \\
\hline$N_{l}$ & Número de ciclos necessários para o inicia da liquefação \\
\hline$N_{m}$ & Contagem do número de golpes do ensaio SPT medido no campo \\
\hline$N_{1-60}$ & Contagem do número de golpes do ensaio $S P T$ normalizado \\
\hline$N_{l-60 C S}$ & $\begin{array}{l}\text { Contagem do número de golpes do ensaio } S P T \text { normalizado para } \\
\text { areia limpa }\end{array}$ \\
\hline & Razão de pré-adensamento \\
\hline$p$ & Tensão total média \\
\hline & Tensão efetiva média \\
\hline
\end{tabular}




\begin{tabular}{|c|c|c|}
\hline & $P$ & Onda primária \\
\hline & $P_{a}$ & Pressão atmosférica \\
\hline & $P_{(n)}$ & Função do modelo de Poisson \\
\hline & $q$ & Tensão de desvio \\
\hline & $q_{c 1}$ & Resistência à penetração da ponta do ensaio CPT \\
\hline & $q_{C A}$ & Resistência à penetração da ponta do ensaio CPT para camadas rijas \\
\hline & $q_{C B}$ & $\begin{array}{l}\text { Resistência à penetração da ponta do ensaio CPT para camadas } \\
\text { moles }\end{array}$ \\
\hline & $q_{c l-N c s}$ & Resistência de ponta do ensaio CPT para areias limpas normalizada \\
\hline & QSS & Estado quase permanente \\
\hline & $\mathrm{R}$ & Onda Rayleigh \\
\hline & $r_{d}$ & Coeficiente de redução de tensão \\
\hline & $r_{u}$ & Razão de poropressão (parâmetro do liquefação) \\
\hline & RMSA & Aceleração da raiz quadrada da média dos quadrados \\
\hline & $\mathrm{S}$ & Onda secundária ou de cisalhamento \\
\hline & SASW & Análise espectral para ondas de superfície \\
\hline & $s_{d}$ & Resistência ao cisalhamento médio do solo liquefeito \\
\hline & $\mathrm{SH}$ & Onda cisalhante horizontal \\
\hline & SV & Onda cisalhante vertical \\
\hline & SPT & Ensaio de Penetração Padrão \\
\hline 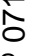 & SSL & Linha do estado permanente \\
\hline & $s_{s u}$ & Resistência ao cisalhamento no estado permanente \\
\hline & $S V$ & Onda cisalhante vertical \\
\hline 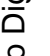 & $s_{u}($ yield $)$ & Resistência não drenada mobilizada na condição de escoamento \\
\hline & $s_{u}($ critical $)$ & Resistência não drenada crítica \\
\hline & $s_{u}(L I Q)$ & Resistência ao cisalhamento na zona liqüefeita \\
\hline & $t$ & Tempo \\
\hline & $T$ & Período do sistema \\
\hline 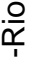 & $t_{f}$ & Duração do sismo \\
\hline & $\tau_{s}$ & Tensão cisalhante estática \\
\hline & $\tau_{c y c}$ & Tensão cisalhante cíclica \\
\hline & $\tau_{c y c, L}$ & Resistência à liquefação cíclica (CRR) \\
\hline & $\ddot{u}_{g}(t)$ & Aceleração do sismo \\
\hline & $v_{p}$ & Velocidade da onda primária \\
\hline & $v_{s}$ & Velocidade da onda de cisalhamento \\
\hline & $v_{s 1}$ & Velocidade da onda de cisalhamento corrigida por sobrecarga \\
\hline & $W$ & Peso \\
\hline & $x$ & Deslocamento relativo \\
\hline & $x$ & Velocidade relativa \\
\hline & $Y$ & Parâmetro do movimento do terreno \\
\hline & $z$ & Profundidade \\
\hline & $\sigma^{\prime} 3 c$ & Tensão confinante efetiva menor \\
\hline & $\sigma_{3}^{\prime}$ & Tensão confinante efetiva \\
\hline & $\sigma^{\prime}{ }_{v c}$ & Tensão efetiva vertical inicial \\
\hline & $\alpha$ & Razão de tensão cisalhante estática inicial \\
\hline & $\alpha_{\sigma \varepsilon}$ & Direção das tensões principais \\
\hline
\end{tabular}


$\psi \quad$ Parâmetro de estado: Ângulo de dilatância

$\xi \quad$ Amortecimento

$\rho \quad$ Massa específica

$\lambda \quad$ comprimento de onda

$\varphi_{m} \quad$ Ângulo de atrito mobilizado

$\varphi_{c v} \quad$ Ângulo de atrito sob volume constante

$u_{\text {excess }} \quad$ Excesso de pororessão (também chamado de $u_{e}$ ) 